\title{
La investigación en las artes universitarias por medio de fuentes primarias.
}

El propósito de este artículo es mostrar el uso de las fuentes primarias para la investigación histórica de las artes en la Universidad de Sonora. Se trata de las Actas de Sesiones del Comité Administrativo de la Universidad de Sonora (CAUS), así como de la Mesa Directiva (MD) ${ }^{1}$ del mismo Comité, localizadas en el catálogo digital del Archivo Histórico de la Universidad de Sonora. Como tal, es un extracto de un ensayo de mayor extensión donde se tratan con más profundidad los comienzos de la educación artística en la institución, por lo cual aquí solo se esbozan algunos fragmentos de esas fuentes y se presenta parte de la información contenida en ellas. A partir de una convención cronológica he dividido el artículo en dos partes: La primera de ellas aborda los orígenes de la Universidad y la segunda, los orígenes de "una escuela de dibujo y pintura". Es, por tanto, un articulo descriptivo que nos permite observar a través de las fuentes consultadas algunos aspectos de la historia universitaria que espera en

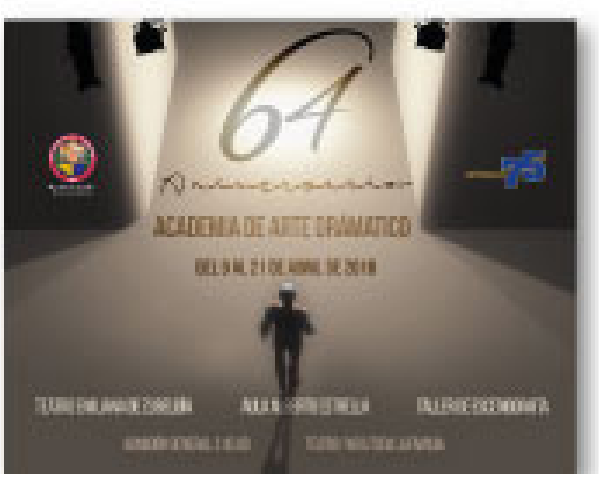

Arturo Valencia Ramos arturo.valencia@unison.mx

Universidad de Sonora

sus repositorios ser investigada.

El 5 de abril de 1939, en el Acta 5 del Comité Administrativo de la Universidad de Sonora el Licenciado Francisco Duarte Porchas en su calidad de Consejero Técnico señalaba:

Queremos que se haga la Universidad, queremos hacerla, debemos hacerla y hacerla pronto y bien y para ello es necesario un dirigente, un cerebro, un orientador, un guía, un constructor, y teniéndolo como lo tenemos entre nosotros, en José Vasconcelos ${ }^{2}$, debemos nombrarlo, conscientes de que al hacerlo, la opinión de todas las gentes de Sonora y de la República entera, descansará confiada[...] Que las generaciones futuras, que van a educarse en nuestra Universidad, no puedan arrojar sobre los miembros del actual Comité Administrativo y sobre sus Consejeros Técnicos, el doloroso pero justiciero cargo de que teniendo entre nosotros a un José Vasconcelos, no supimos aprovecharlo en la realización

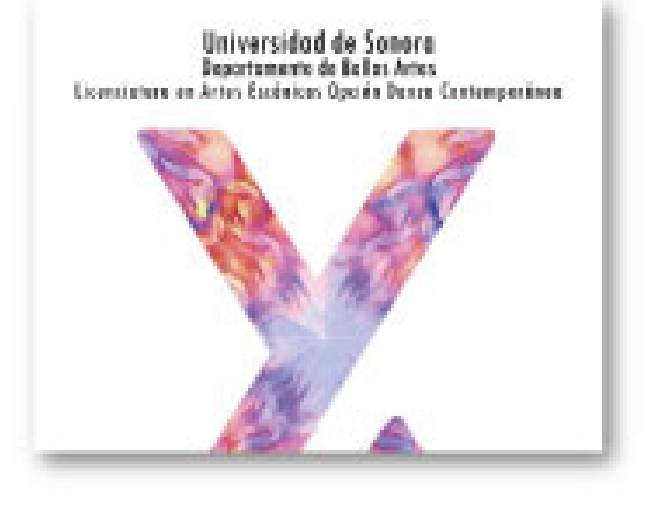




\section{(Arte)}

de la más alta, de la más útil y noble tarea que se ha emprendido hasta hoy en Sonora: la creación de la Universidad (Acta 5 del CAUS).

Durante esa misma sesión "El señor [Felipe] Seldner [Consejero Técnico del CAUS] manifiesta que se ha opuesto y se opone a la designación propuesta en favor del licenciado Vasconcelos por considerar que dicho señor, aún cuando tenga el propósito de estar retirado de la política, seguirá teniendo ante el público el carácter de político por toda su actuación anterior" (Acta 5 del CAUS).

\section{El 23 de mayo de 1939:}

Se dio cuenta en seguida (sic) con la comunicación del señor licenciado Vasconcelos que delinea a grandes rasgos el programa de estudios de la Universidad. Se cambiaron impresiones en lo general sobre la conveniencia de estudiar la impantación (sic) de carreras que estén acordes con las necesidades prácticas de la economía regional, así como con el caracter (sic) de la población de esta zona (Acta 3 de la Mesa Directiva del CAUS).

La historiografía sonorense y en particular la universitaria tiene todavía un importante pendiente en cuanto al estudio del conflicto que generó la presencia de José Vasconcelos como primer or- ganizador de la Universidad de Sonora. Como sabemos, Vasconcelos quedó fuera del proyecto y no fue sino hasta tres años después que se tiene noticias suyas en el CAUS. El 23 de septiembre de 1942, el Dr. Domingo Olivares recibió una carta de José Vasconcelos:

...ofreciéndole su colaboración amplia para la Universidad de Sonora. Semejante actitud de dicho abogado, - comentó el Dr. Olivares-, revela que no existen rencores y que quedaron definitivamente liquidadas las divergencias de criterio que durante los primeros meses de actuación del Comité se suscitaron entre algunos de sus miembros y el letrado a que se

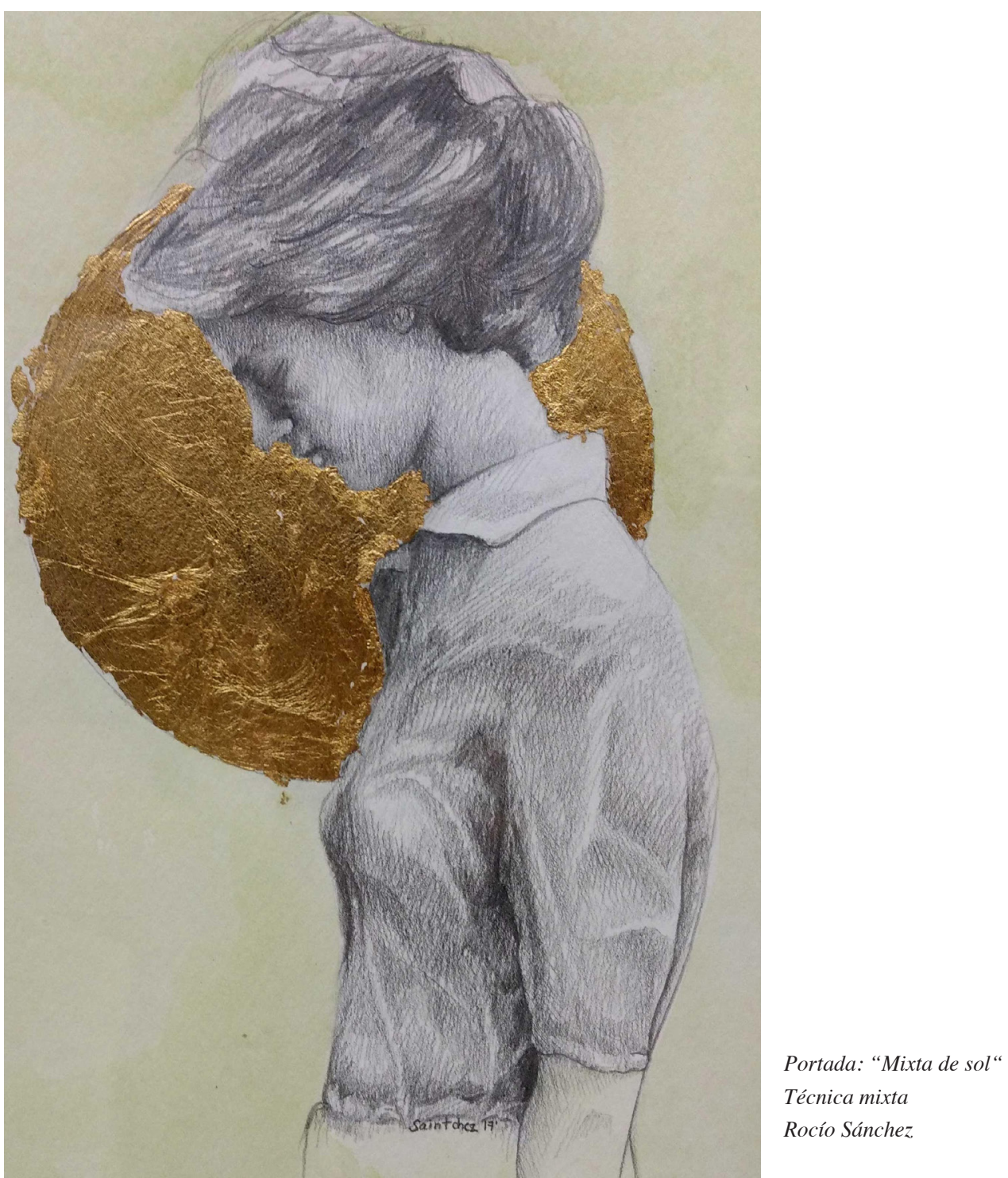


hace mención. Además,_-dijo-, cabe admitir que la satisfacción manifestada por el Lic. Vasconcelos es sincera, porque se trata nada menos que de la realización de su idea (Acta 21 de la Mesa Directiva del CAUS).

Los trabajos no se detuvieron, sin embargo, y el 13 de junio de 1941 se asentaban en el acta 8 de la MD del CAUS las condiciones y posibilidades de iniciar la educación universitaria con la incorporación a la institución en formación de las escuelas secundaria, normal y preparatoria. Opinó el Presidente de la MD que "Son necesarias porque la primera capacitará a los alumnos para emprender sus estudios de Preparatoria y la segunda vendrá a llenar una urgente necesidad planteada desde hace mucho tiempo por la escasez de buenos maestros de escuela en el Estado" (Acta 8 MD CAUS, 13 de junio de 1941). Desde los inicios de los trabajos se habían establecido relaciones con los ganaderos organizados a fin de que aportaran recursos económicos para la realización del proyecto universitario dado que se trataba de establecer carreras acordes a la economía regional. Esos recursos estaban estimados para las edificaciones en $\$ 400,000.00$ pesos (Acta 9 MD CAUS, 3 de julio de 1941). Igualmente ya desde 1941 se proponía la creación de Facultad de Agricultura de la Universidad de Sonora (Acta 10 MD CAUS, 20 de septiembre de 1941), misma que no se realizaría

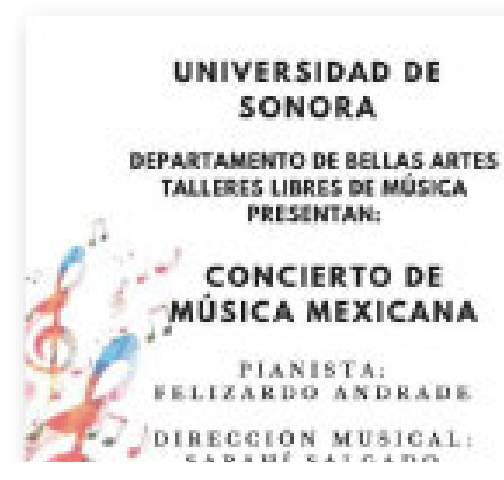

sino hasta la década de los años 1950. La incorporación de los secundarianos, preparatorianos y normalistas era una cuestión estratégica dado que con ello se pretendía garantizar la existencia de estudiantes hacia los cuales orientar la educación universitaria para los corto y mediano plazos.

En cuanto a la educación artística en la Universidad de Sonora, los primeros registros que se tienen quedan consignados en el acta 17 de la Mesa Directiva. Dentro de todos los temas a discusión centrados básicamente en los aspectos económicos y operativos de la construcción de los edificios universitarios, el consejero Rafael Treviño introduce el tema como necesario dado el perfil de los estudiantes con los cuales iniciaría la naciente institución.

A continuación el señor Rafael Treviño solicitó autorización de la Mesa Directiva para que se lleve a cabo la compra de instrumentos para una banda de música que, en su concepto, debe formarse por alumnos de la Escuela Secundaria y Normal, con el propósito de despertar en ellos el amor por el arte musical y de fomentar su confraternidad mediante la sana alegría que el conjunto proporcionará (Acta 17 MD CAUS, 23 de abril de 1942).

La propuesta fue aprobada por cinco votos contra dos. Quienes votaron en contra no consideraban "muy necesaria la banda de música".

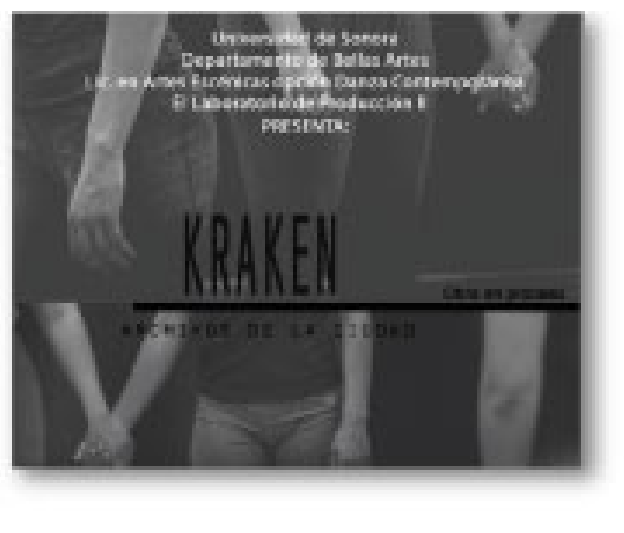




\section{(Arte)}

Para 1944 ya se había organizado a los profesores universitarios en el Seminario de Cultura con las secciones de humanidades, ciencias físico-químicas y matemáticas, ciencias biológicas, ciencias sociales, actividades estéticas y actividades prácticas. En ese seminario participaba el profesor Francisco Castillo Blanco.

El 11 de junio de 1948 el rector Manuel Quiroz Martínez comunica a la MD del CAUS su informe sobre la creación de una Escuela Libre de Artes Plásticas. El proyecto lo propuso el 23 de febrero de ese mismo año el profesor Francisco Castillo Blanco quien ya tenía una experiencia docente en la ciudad desde 1935 y a quien se le asignó dibujar el escudo universitario (Acta 62 MD CAUS, 26 de abril de 1945.) .

Su proyecto proponía la creación de la Escuela Libre de Artes Plásticas, con el objeto de "fomentar y elevar el sentimiento estético en nuestro medio" y aplicar en la industria y comercio los conocimientos que se impartieran dado que "las Artes Plásticas no son ajenas a estas ramas de nuestra vida activa". Los beneficios anímicos incluidos en el proyecto resultarían en un mejoramiento de las ideas, el desarrollo de la imaginación, la ampliación del sentido de observación y la formación de un espíritu creativo: "Existen facultades estéticas que debidamente orientadas redundarían en magníficos artistas, y si hasta la fecha se han malogrado esos elementos ha sido por la falta de medios, un apropiado lugar para el estudio y falta de estímulo".

El profesor Castillo Blanco proponía resolver con ello el problema de "una realidad no despreciable en esta época de enfermedades espirituales creadas por nuestra vida mecanizada." El Rector, después de analizar el proyecto, informa a la MD del CAUS que es necesario impartir conocimientos artísticos en esta zona del país. Ya con anterioridad la institución "había venido pugnando por la creación de escuelas o departamentos universitarios, encargados de difundir conocimientos sobre artes mayores y despertar sentimientos de interés y amor por las ramas estéticas, como condición necesaria para proporcionar una cultura integral a la comunidad (FUS/SOG/MDCAUS/S01/C2. Exp. 10. Leg 2-4).

El Rector aceptaba la propuesta aunque condicionada a un plan máximo de dos años, contar con un mínimo de alumnos así como con el profesorado idóneo y asegurar la capacidad económica de la Universidad para su sostenimiento. El carácter "libre" de la escuela implicaba como condición tener solo la instrucción primaria terminada y que no se exigiera "ninguna otra preparación cultural previa", ya que en caso de imponer la condición de la enseñanza secundaria-decía el rector-no habría alumnos dado que quienes terminan su ciclo secundario "prefieren carreras de especulación científica o económica y no otras".

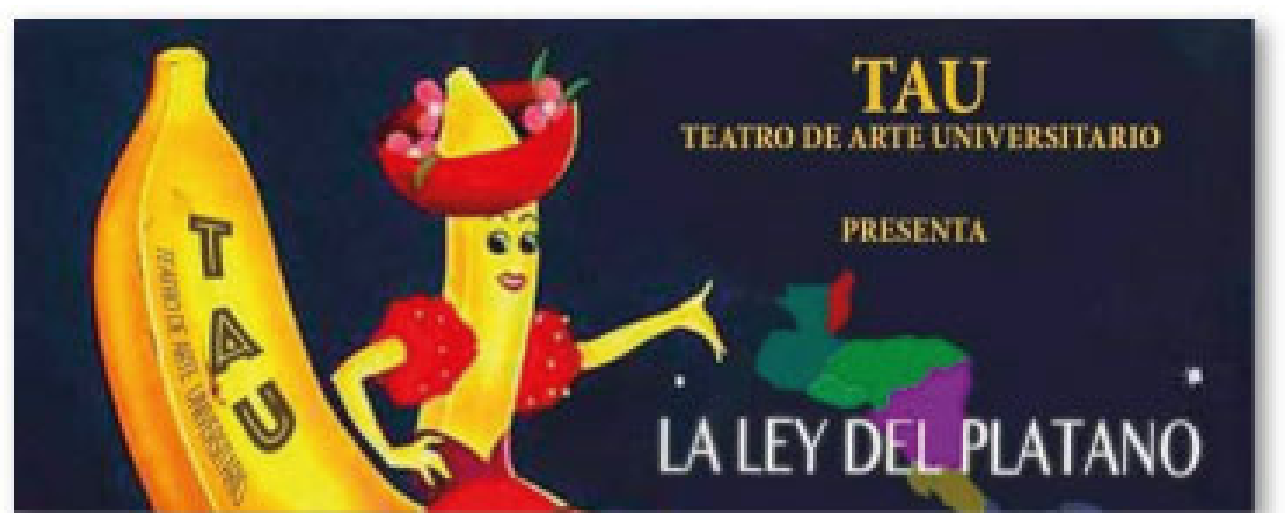


La condición de "libre" implicaba también que la escuela debía ser nocturna ya que "si fuese diurna tendría que tomar las características de un plantel con exigencias básicas regulares, requisitos que más alejarían su posibilidad de contar con alumnos". La propuesta de Castillo Blanco incluía a un maestro pintor, a un escultor y a un fotógrafo con tres horas de trabajo diario para cada uno, "proposiciones buenas para una escuela diurna; pero impracticables en una escuela nocturna"-según la apreciación de Quiroz Martínez. El presupuesto global de salarios al año fue calculado en 7,920 pesos más 5,000 pesos de equipamiento por una sola vez por lo que no resultaba viable para las finanzas universitarias. De esta forma la recomendación del rector fue "dar un nuevo arreglo a la iniciativa a fin de que la citada Escuela pueda ser nocturna y su costo pueda reducirse al mínimo." Pasaron dos años. El 23 de diciembre de 1950, el Dr. Ignacio Cadena, Presidente del CAUS escribe al profesor Manuel
Quiroz Martínez, Rector de la Universidad de Sonora, que el 20 de diciembre la Mesa Directiva había acordado abrir para enero de 1951 la nueva escuela Libre de Pintura y Dibujo:

...el Doctor Cadena comunica que uno de los deberes del Comité de la Universidad es crear escuelas que vengan a llenar necesidades culturales y para ello presenta un proyecto y es el que se establezca una escuela de dibujo y pintura; para llevarlo a cabo se ha apalabrado con el Artista y pintor señor Don Higinio Blat, español de grandes méritos quien por motivos de salud se ha radicado en nuestra ciudad; por las recomendaciones que trae demuestra que conoce y sabe enseñar ese arte; que le pidió un programa de enseñanza el cual llevo para su estudio con el señor Rector y que a la vez estudiara la posibilidad de llevarlo a la práctica...(Acta 138 de la MD del CAUS, 20 de diciembre de 1950).

Los cursos se iniciaron en enero de 1951.

\begin{abstract}
${ }^{1}$ El Comité Administrativo de la Universidad de Sonora (CAUS) fue constiuido de acuerdo a lo dispuesto en el Título Primero Capítulo Primero de la Ley No. 92 de enseñanza universitaria, aprobada el 22 de noviembre de 1938. Las sesiones del CAUS se celebraban mensual y anualmente; eran convocadas por la Mesa Directiva y ante ella se presentaban los informes correspondientes de los trabajos llevados a cabo [...]Cabe hacer mención, que en los quince años que trabajó el CAUS y la Mesa Directiva se construyeron el edificio de Rectoría y los de las primeras escuelas: secundaria y preparatoria. Se establecieron las escuelas de Iniciación Universitaria que funcionaba paralelamente con la secundaria, pero fue suprimida en 1946, y las escuelas de Enfermería, Farmacia, Ingeniería y de Comercio. Además, estaba en proceso la construcción de la Escuela de Agricultura y Ganadería [...] Del periodo 1938-1953, la presidencia del CAUS estuvo presidida por el Dr. Domingo Olivares (1938-1944); Ing. Ramón Corral (1944-1949) y Dr. Ignacio Cadena H. (1949-1953). http://www.archivohistorico.uson.mx/into/servicios/Actas\%20de\%20sesiones\%20del\%20Comit\%C3\%A9\%20Administrativo. htm
\end{abstract}

${ }^{2} J o s e ́$ Vasconcelos (Oaxaca, 1882- Cd., de México, 1959). Abogado, filósofo, político, escritor. Rector de la UNAM, 1920-1921; Fundador de la Secretaría de Educación Pública durante el régimen presidencial de Álvaro Obregón, 1920-1924; Candidato presidencial en 1929; Primer organizador de la Universidad de Sonora en 1939.

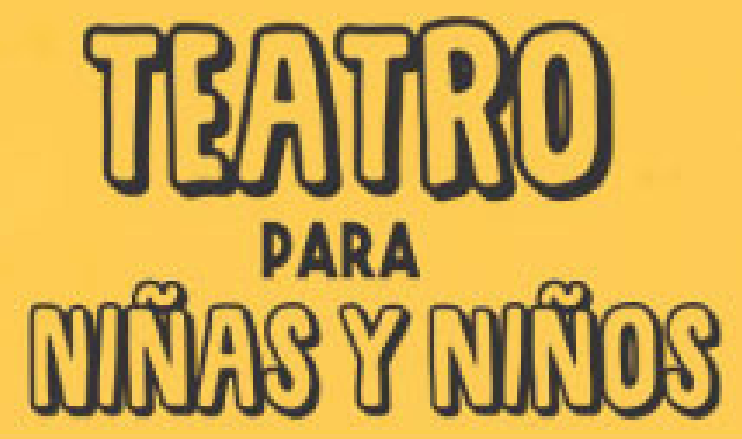




\section{(Arte)}

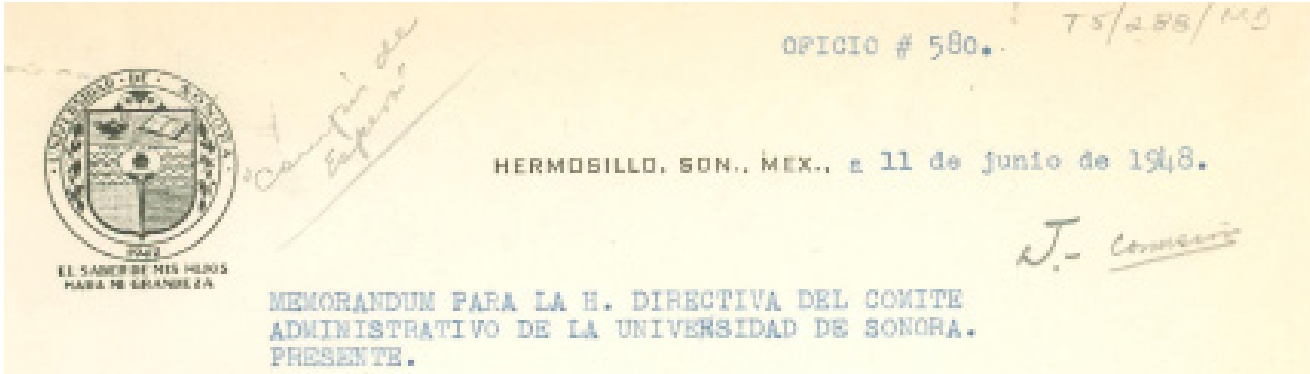

Asunto: Cregolón de un Bacueln Libre de Artes Pláatiosas.

Com1sionada esta Rectorla psrs oxnm1nnr la inle1et1va que sobre in fundeclón de una Bgcuela de Artas plísticas, que sbaros Plntura $y$ Bsculture, fué presentadg por el sefior Prof. Pranclaco Cost1110 Blanco, ls propd a Rectorín presente lns -. sigulentes conelusiones:

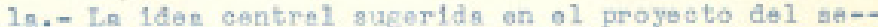

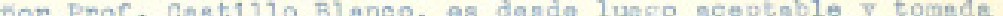

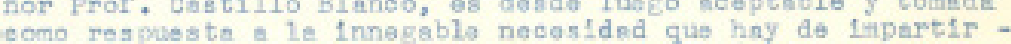
conoolmiantos artiaticos on osts zonn del pals.

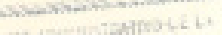
nte wathy

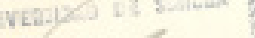

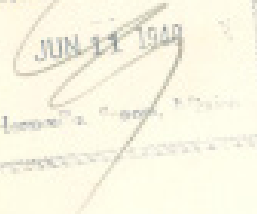

2n, - Esta Inst1tuofón, de tlempo ntrís, ha venido pug pando por in creación de escuelan o departamentos universitsrlos, encersados de difundir conoelimlentos sobre artes mayores

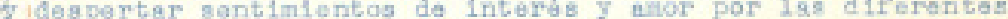

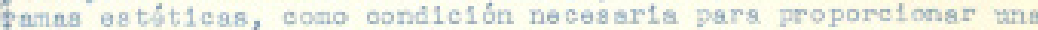
dultura integrei a la coatunldad.

Por este razín, en prinolplo, in Rectorle acoge con interés la Inlelative del soñor Prof. Cast1110 Blanco.

31n ombareo, como on Gesos análogos, In visbilidad de osta Iniclativa tiene que a fustargo a las algulentes condiclo nes bésices:

a) Batrechar au desnrrolla un plan minimo mís reducldo suin que coto se proponen en la 1 niolativn.

b) Contsir pravinmente con un número mínimo de slumos

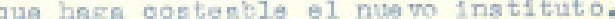

c) Contar con profesorado 1 dóneo.

a) Asegurarse de $i_{a}$ capacidad econón1eg de $I_{Q}$. Jn1vera1ded pars gastoner el presupuesto de osta nuevn empreas.

e) Considersr ol costo de equipos que se requiern.

En relactón con estas elnco cond1c1ones, ln Rectorla

oplne:

10.- Que de sprobarse 1a In1e1ativa dol Sr. Frof. Cast1110 Blanen, su plan de netividades y estudlos, Ceberá abor-cor un tienpo máximo de dos effos, cono lo suglere el propononte.
Fuente Primaria: Documento de Archivo Histórico

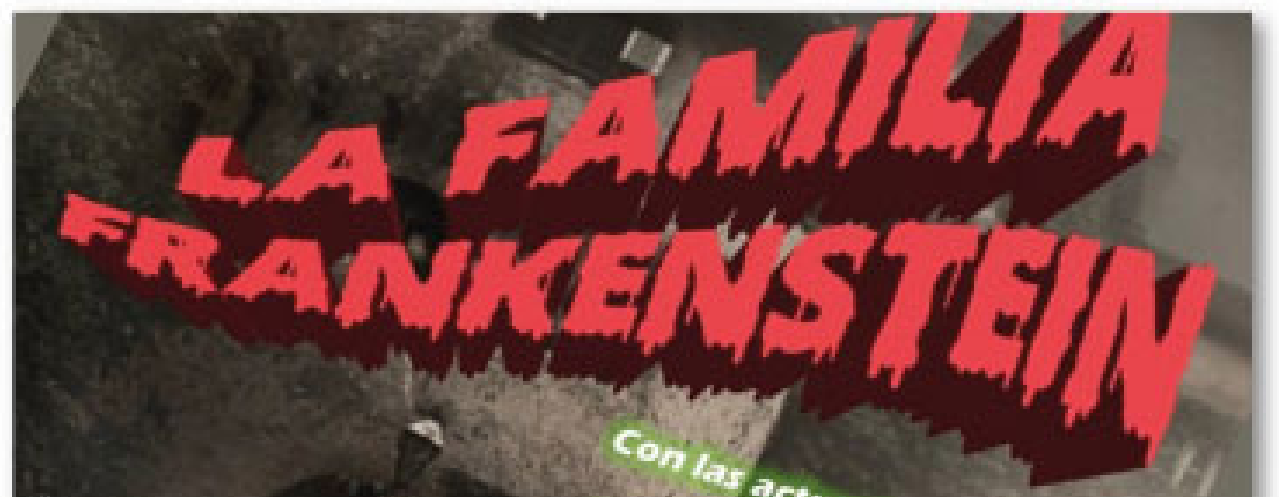




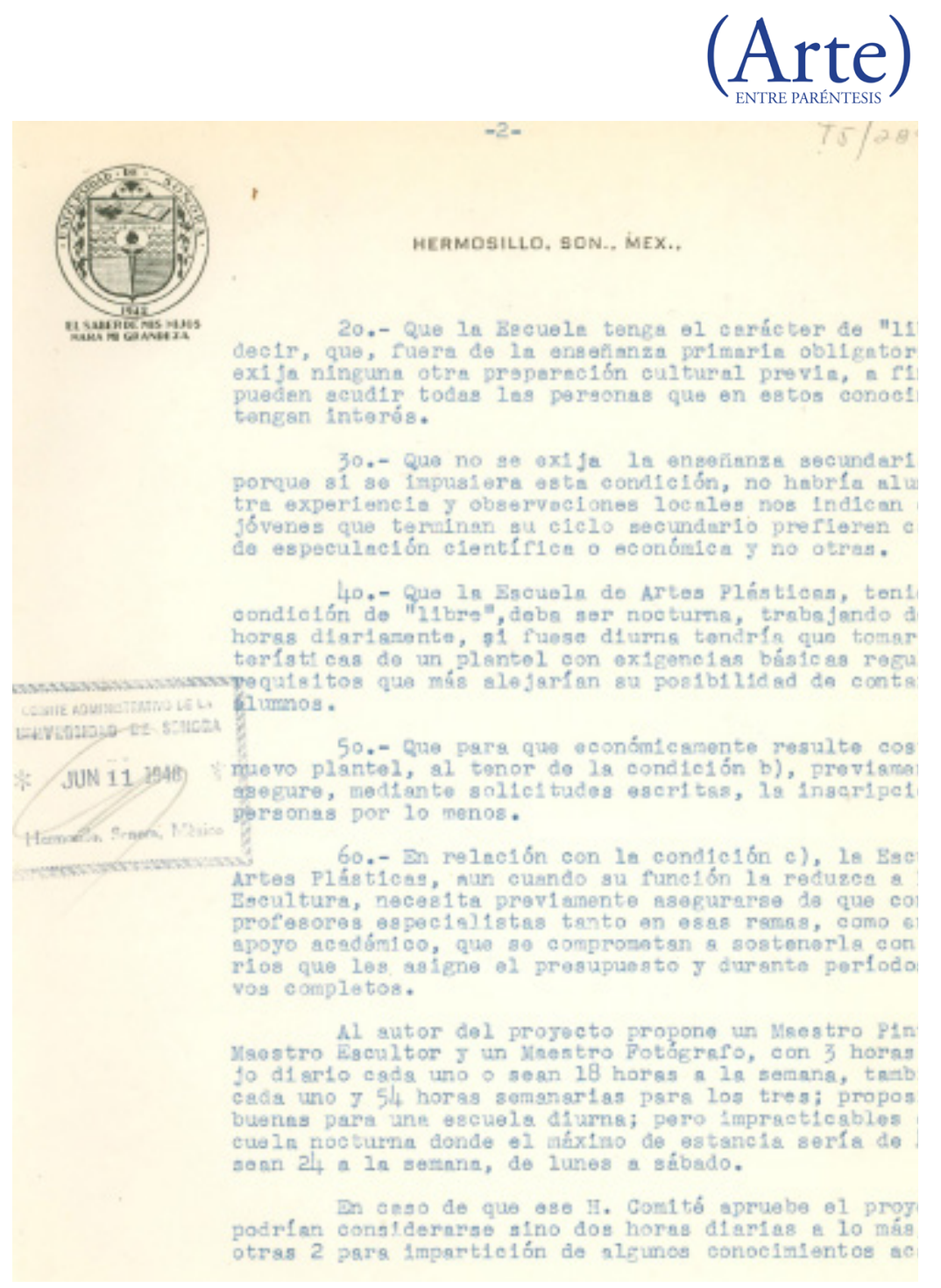

Archivo Histórico

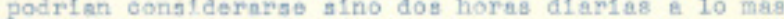

Fuente Primaria: Documento de

Fuente Primaria: Documento de

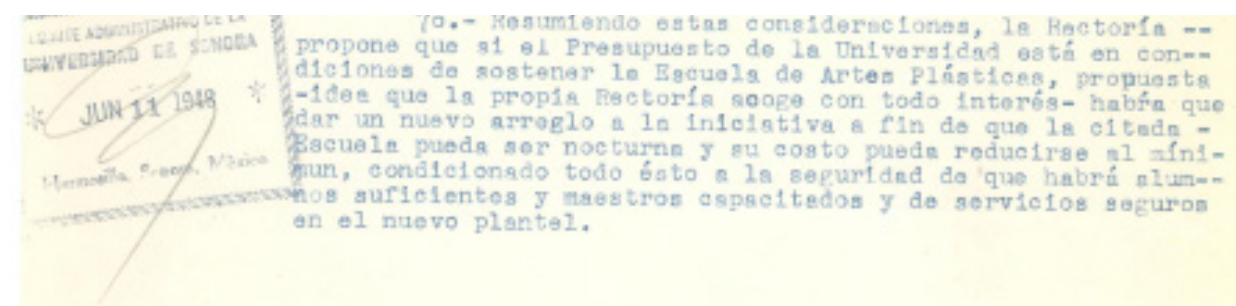

NUY ATEMTAMBHTE.

"BL. SAFER DE IIS HIJOS HARA MI GRANDBZA".

Archivo Histórico

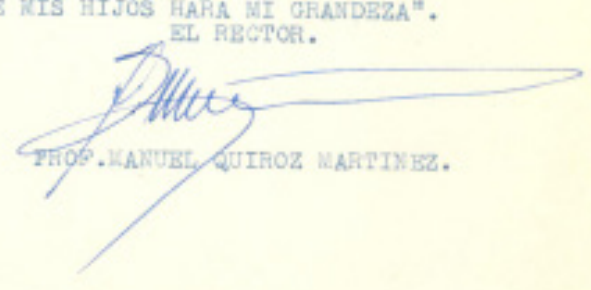

